\title{
Mucormycosis of Maxilla Post Covid-19-An Epidemic in India-Report of a Case
}

\section{Gokul Venkateshwar ${ }^{1}$, Tanvi Mahale ${ }^{2 *}$, Veenita Singh ${ }^{3}$ and Deepak Khanna $^{4}$}

${ }^{1}$ Professor Department of Oral and Maxillofacial Surgery, D Y Patil University

School of Dentistry, Navi Mumbai, Maharashtra, India

${ }^{2}$ Resident, Department of Oral and Maxillofacial Surgery, Navi Mumbai,

Maharashtra, India

${ }^{3}$ Professor, Department of Oral and Maxillofacial Surgery, D Y Patil University

School of Dentistry, Navi Mumbai, Maharashtra, India

${ }^{4}$ Consultant Oncosurgeon, D Y Patil Hospital and Research Centre, Navi Mumbai,

Maharashtra, India

*Corresponding Author: Tanvi Mahale, Resident, Department of Oral and

Maxillofacial Surgery, School of Dentistry, Navi Mumbai, Maharashtra, India.
Received: May 20, 2021

Published: June 12, 2021

(C) All rights are reserved by Tanvi Mahale., et al.

\section{Abstract}

This article presents a case of a 41-year-old, male patient affected with Mucormycosis of the right maxilla following COVID-19 infection. Though Mucormycosis has been reported in the literature the condition has exponentially increased following COVID-19 pandemic due to a plethora of factors.

Keywords: Mucormycosis; Amphotericin B; COVID-19 Infection; Immunocompromised

\section{Abbreviations}

OPG: Orthopantomogram; CT Scan: Computed Tomography Scan; HRCT: High Resolution Computed Tomography; COVID-19: Corona Virus Disease of 2019; CBC: Complete Blood Count; ESR: Erythrocyte Sedimentation Rate; PPBS: Post Prandial Blood Sugar; FBS: Fasting Blood Sugar; LFT: Liver Function Test; RFT: Renal Function Test

\section{Introduction}

Mucormycosis of the maxilla is a rare but a debilitating infection that can affect immunocompromised patients in healthcare settings or even otherwise. Investigations of possible Mucormyco- sis outbreaks in healthcare settings and the outbreak in the post COVID-19 phase has lead to identification of possible potential causes [1]. Mucormycosis is caused by fungi of order Mucorales, recent classification has placed Mucorales in the order subphylum Mucormycotina [13] and among the Mucoraceae, Rhizopus oryzae (Rhizopus arrhizus) is the causative cause of infection [7]. Based on the general clinical presentation Mucormycosis can be divided into six clinical categories 1) rhinocerebral 2) pulmonary 3) cutaneous 4) gastrointestinal 5) disseminated 6) miscellaneous. Since the advent of novel strain of severe acute respiratory syndrome coronavirus 2 (SARS CoV-2) the healthcare system has been overwhelmed and burdened in containing and treating this new virus strain [5,6]. 
Among the Post covid complications, the incidence of invasive fungal infections (IFI) of the maxillofacial region, namely Mucormycosis and aspergillosis has increased exponentially. Though the most common type of Mucormycosis is the rhino-cerebro-orbital type which happens when spores are inhaled into the paranasal sinuses of susceptible hosts, we are witnessing Mucormycosis of the maxilla in various manifestations with extensions into the orbit and nose [4]. Mucormycosis is seen typically in the immunocompromised patients among the various predisposing conditions are Diabetic Ketoacidosis, malnutrition etc [7]. The clinical hallmark is vascular invasion resulting in thrombosis and tissue infarction necrosis [7]. The typical presenting signs and symptoms are facial cellulitis, headache, proptosis, mobile teeth, discharging sinus [12].

\section{Case Presentation}

A 41-year-old, male patient presented with a chief complaint of mobile teeth and pus discharge in the upper right region of the jaw to Department of Oral and Maxillofacial Surgery, D.Y Patil University School of Dentistry, Navi Mumbai.

Past medical history-The patient was hospitalised for COVID-19 one and a half months earlier and was in the ICU among the drugs administered was a high dose of steroids. Also had history of dengue a month before COVID-19.

\section{Clinical features:}

Facial findings (Figure 1-3).

Facial swelling on the right side of the face.

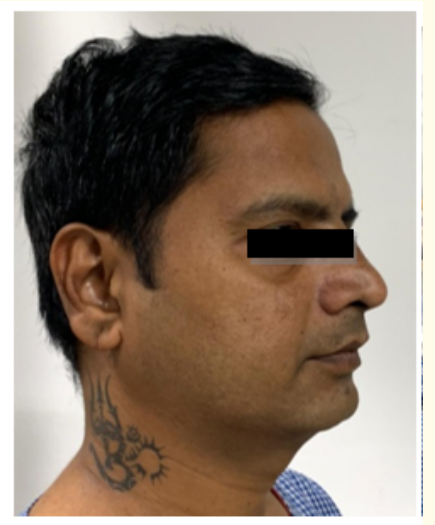

Figure 1

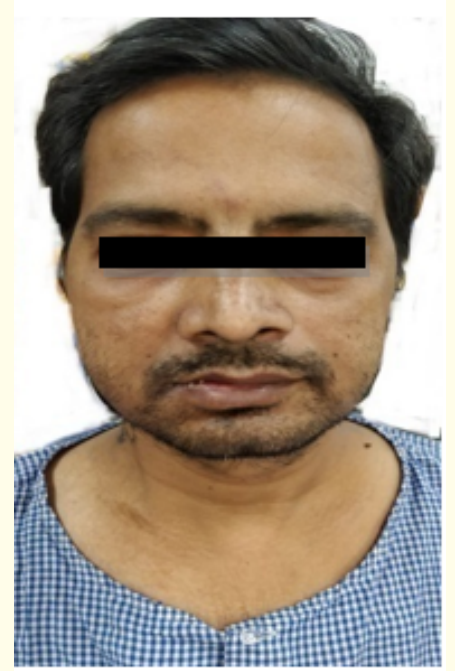

Figure 2

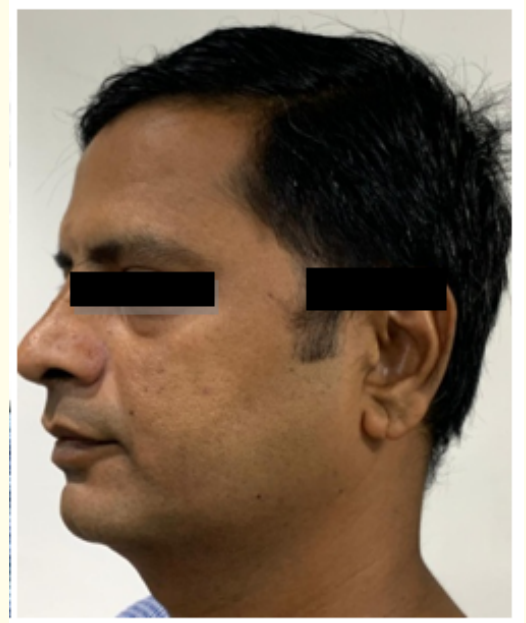

Figure 3

\section{Intraoral findings}

Clinical examination revealed mobility of right maxilla, severe mobility of teeth from maxillary central incisor to maxillary right third molar, Intraoral pus discharge, Ulceration of mucosa, sinus tract present between maxillary right lateral incisor and canine bucally, carious maxillary right first molar, right maxillary buccal and palatal bone expansion. 


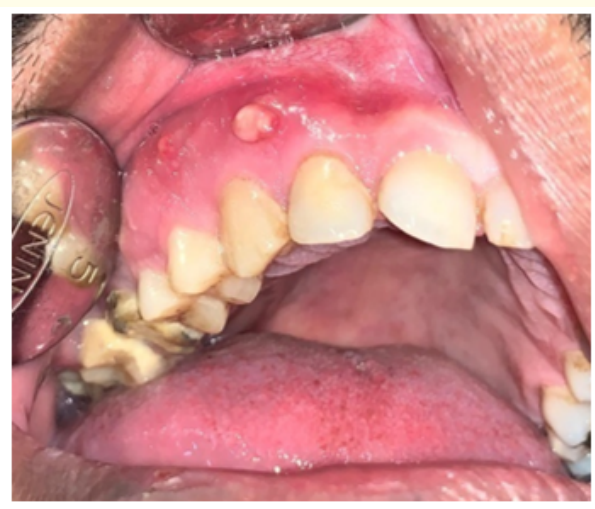

Figure 4: Intraoral right maxillary buccal and palatal cortical expansion with sinus tract.

Routine laboratory investigations were done prior to surgery Imaging

An OPG and a CT scan (With contrast) and plain was done.

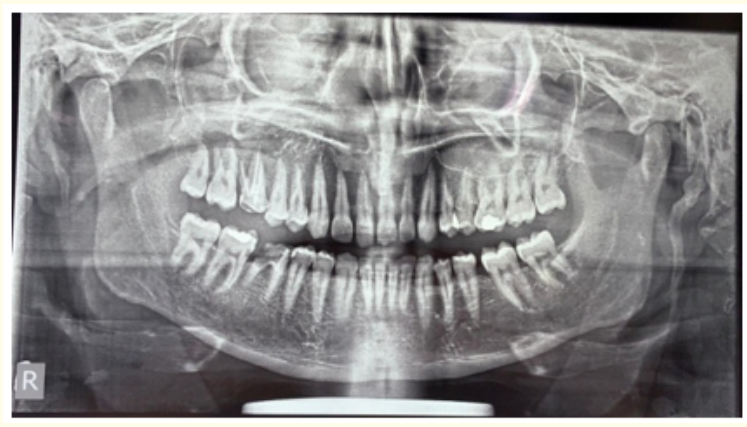

Figure 5: Pre-operative OPG.

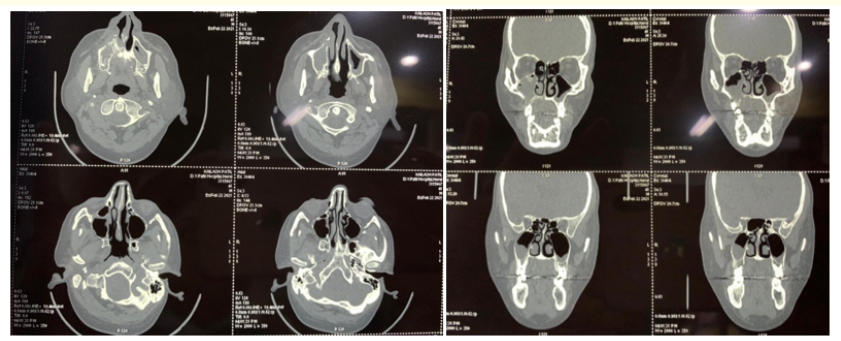

Figure 6: CT face showing axial and coronal views.
OPG showed bone loss in the right upper alveolus.

\section{CT scan findings (Figure 6)}

- Erosion and thinning of hard tissues enlargement of masticatory muscle.

- Mucosal thickening of sinuses changes in fat planes.

- Obliteration of right maxillary sinus.

Intraoral incisional biopsy was done confirming Mucormycosis.

\section{Treatment}

The successful treatment of Mucormycosis requires four steps: 1) early diagnosis; 2) reversal of underlying predisposing risk factors, if possible; 3) surgical debridement/debulking where applicable and 4) prompt antifungal therapy [7].

Pre-operative management

- Intravenous pre-operative antibiotics were administered.

- Inj Amphotericin B $40 \mathrm{mg}$ (conventional) was given intravenously with renal monitoring and increase in serum creatinine levels $(4.2 \mathrm{mg} / \mathrm{dl})$ was seen within 48 hours. Conventional Amphotericin was stopped and Liposomal Amphotericin $80 \mathrm{mg}$ four times a day was started after consulting the Nephrologist to avoid further renal insult. The patient was taken up for surgery once the values were acceptable.

\section{Surgical procedure (Figure 8-11)}

The debridement of the lesion was done under General Anesthesia after following the surgical protocols. The lesion was accessed trans orally and the affected maxilla including the palate till the mid palatal region, superiorly sparing the infraorbital foramen, posteriorly till the zygoma (buttress) anteriorly till the naso palatine foramen were excised. The entire sinus lining was curetted till fresh bleeding was seen. The wound was closed primarily. Nasal antrostomy was performed.

\section{Post-operative management}

Inj Liposomal Amphotericin B was administered intravenously 10 days post op and patient was discharged thereafter. He is currently asymptomatic and comes for regular follow up. Also an obturator is being planned. 


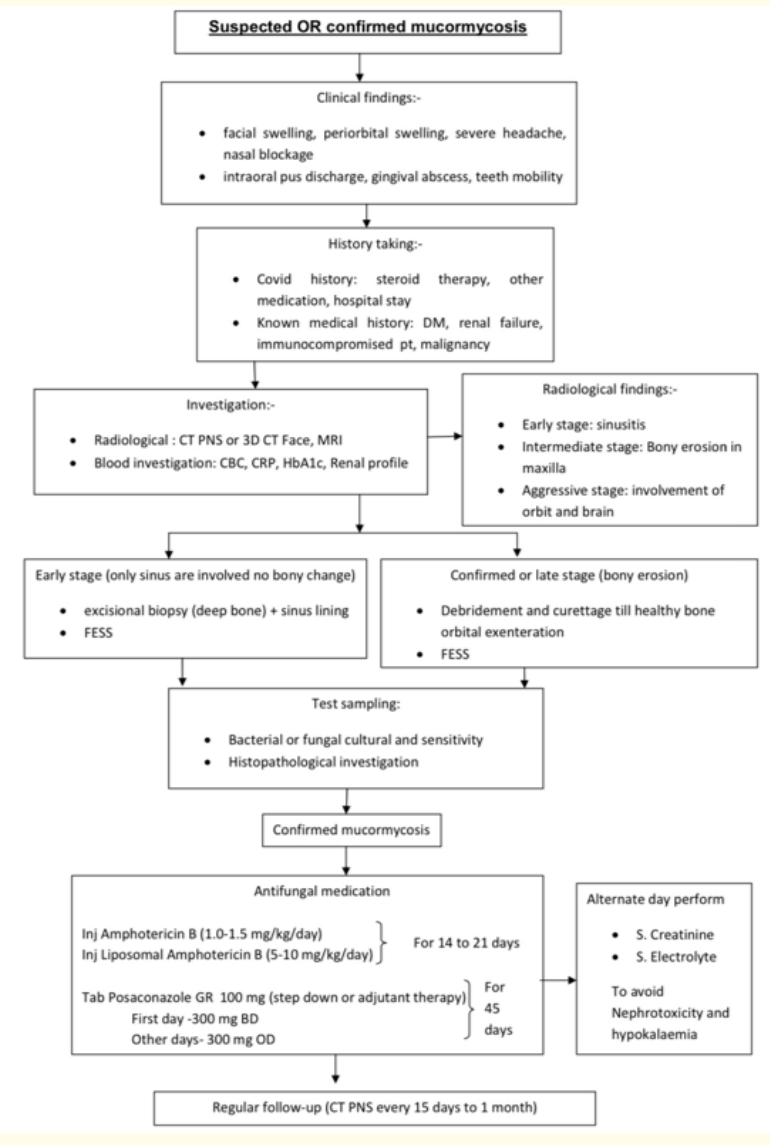

Amphotericin B administration protocol

\begin{tabular}{|c|c|c|}
\hline Drugs & Recommended Dose & Duration \\
\hline Amphotericin B & $1.0-1.5 \mathrm{mg} / \mathrm{kg} /$ day & $\begin{array}{c}14 \text { to } 21 \text { days } \\
\text { depending on } \\
\text { severity }\end{array}$ \\
\hline $\begin{array}{c}\text { Liposomal Amphotericin } \\
\text { B }\end{array}$ & $5-10 \mathrm{mg} / \mathrm{kg} /$ day & \\
\cline { 1 - 2 }
\end{tabular}

Figure 7: Protocol for mucormycosis.
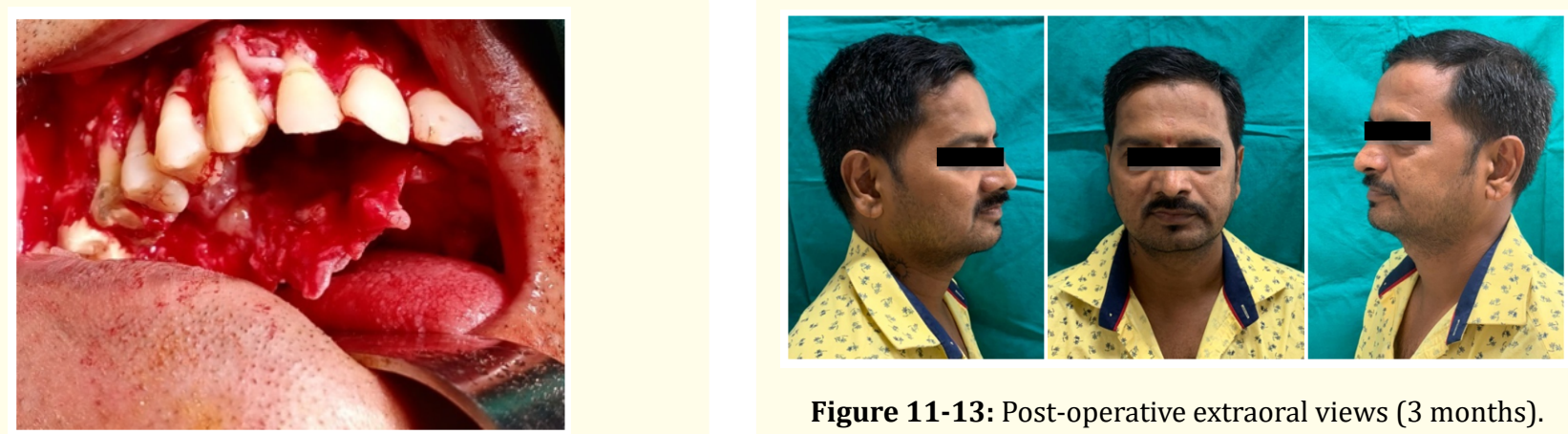

Figure 11-13: Post-operative extraoral views (3 months).

Figure 8: Exposure of the lesion. 


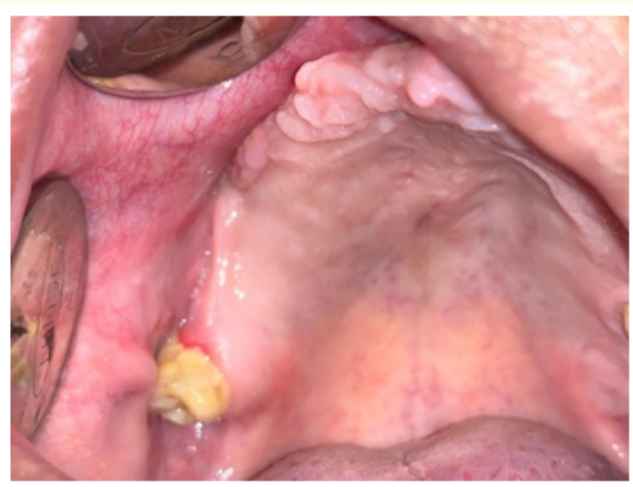

Figure 14: Post-operative intra oral healing (3 months).

\section{Discussion}

There are specific pathophysiologic features of COVID-19 that may permit secondary fungal infections, including a propensity to cause extensive pulmonary disease and the subsequent alveolointerstitial pathology that may enhance the risk of invasive fungal infections. Second, the immune dysregulation associated with COVID-19, with reduced numbers of T lymphocytes, CD4+T and CD8+T cells, may alter innate immunity [9]. A complex interplay of factors, including pre-existing conditions such as diabetes mellitus, previous respiratory pathology, use of immunosuppressive therapy, the risk of hospital-acquired infections, and systemic immune alterations of COVID-19 infection itself may lead to secondary infections, which are increasingly being recognized in view of their impact on morbidity and mortality [10]. Mucormycosis treatment recommendations were recently updated by the European Confederation of Medical Mycology. Although Amphotericin B deoxycholate was the cornerstone of Mucormycosis therapy for decades lipid formulations i.e. liposomal Amphotericin B can be administered safely in higher doses for a longer period than Amphotericin B, being less nephrotoxic and now is used as the first-line drug in Mucormycosis therapy [13]. Isavuconazole and new Posaconazole formulations have been added to the guidelines but in second-line treatment following Liposomal-Amphotericin B due to some remaining issues. Few anti-Mucorales drugs are currently under development. Moreover, evidence for adjunctive therapies is scarce and doubts on their effectiveness persist due to a lack of randomized prospective controlled studies. They are particularly complex to implement in a context of low incidence disease. Few advances have been made on Mucormycosis treatment. However, empowering new concepts and new routes of administration to fight this devastating disease appear promising and are to be encouraged [11].

\section{Conclusion}

Mucormycosis of the maxilla is an hitherto known but obscure fungal disease is now presenting in the post COVID-19 phase in a manner unknown previously. Random and indiscriminate use of corticosteroids, late diagnosis of the disease is increasingly making it difficult to tackle this fungal invasion which happens in a span of few days. However, with better understanding and early detection Mucormycosis can be controlled at an initial stage with a combination of medical and surgical intervention.

\section{Acknowledgements}

Dr. Amit Date, Professor Department of Oral and Maxillofacial Surgery, D Y Patil University School of Dentistry, Navi Mumbai, Maharashtra.

\section{Bibliography}

1. Kathleen P Hartnett., et al. "A Guide to Investigating Suspected Outbreaks of Mucormycosis in Healthcare". Journal of Fungi 5 (2019): 69.

2. Richardson M. "The ecology of the Zygomycetes and its impact on environmental exposure". Clinical Microbiology and Infection 15 (2009): 2-9.

3. Roden MM., et al. "Epidemiology and outcome of zygomycosis: A review of 929 reported cases". Clinical Infectious Diseases 41 (2005): 634-653.

4. Cox G. Mucormycosis (2020).

5. Guan WJ., et al. "Clinical characteristics of coronavirus disease 2019 in China". The New England Journal of Medicine 382.18 (2020): 1708-1720.

6. Goyal P., et al. "Clinical characteristics of Covid19 in New York City". The New England Journal of Medicine (2020).

7. Spellberg B., et al. "Novel perspectives on Mucormycosis: pathophysiology, presentation, and management". Clinical Microbiology Reviews 18 (2005): 556-569. 
8. Mehta S and Pandey A. "Rhino-Orbital Mucormycosis Associated with COVID-19". Cureus 12.9 (2020): e10726.

9. Gangneux JP., et al. "Invasive fungal diseases duringCOVID-19: we should be prepared”. Journal of Medical Mycology 30 (2020): 100971.

10. Chen N., et al. "Epidemiological and clinical characteristics of 99 cases of 2019 novel coronavirus pneumonia in Wuhan, China: a descriptive study". Lancet 395 (2020): 507-513.

11. Brunet $\mathrm{K}$ and Rammaert $\mathrm{B}$. "Mucormycosis treatment: recommendations, latest advances, and perspectives". Journal de Mycologie Medicale (2020).

12. Aditya Moorthy., et al. "SARS-CoV-2, Uncontrolled Diabetes and Corticosteroids-An Unholy Trinity in Invasive Fungal Infections of the Maxillofacial Region? A Retrospective, Multi-centric Analysis (2021).

13. Brad Spellberg and Ashraf S Ibrahim. "Recent Advances in the Treatment of Mucormycosis". Current Infectious Disease Reports 12 (2010): 423-429.

Volume 5 Issue 7 July 2021

(C) All rights are reserved by Tanvi Mahale., et al. 